\title{
MODELAGEM DE INFORMAÇÕES PARA CONSTRUÇÃO (BIM): AMBIENTES COLABORATIVOS PARA GESTÃO DE PROJETOS E OBRAS NA CONSTRUÇÃO CIVIL
}

\author{
Raysa Maria de Souza Gomes ${ }^{1}$, Alice de Assis Abreu ${ }^{1}$, Miguel Aguiar Bauer ${ }^{1}$, Gabriela \\ Carvalho Nascimento ${ }^{1}$, Maurício Soares do Vale ${ }^{2}$
}

\section{RESUMO}

GOMES, R.M.S.; ABREU, A.A.; BAUER, M.A.; NASCIMENTO, G.C.; VALE, M.S. Modelagem de informações para a construção (BIM): ambientes colaborativos para gestão de projetos e obras na construção civil. Perspectivas Online: Exatas \& Engenharia, v.8 , n.23 , p. $48-62,2018$.

No setor da construção civil, a cada dia surgem novas soluções em métodos, ferramentas, processos e conceitos. Seu uso, à medida do possível, permitem as empresas se manterem ativas e competitivas. Depois de apresentar soluções de Enterprise Resources Planning (ERP), a área de Tecnologia da Informação (TI) traz para o setor da construção civil a Modelagem da Informação da Construção (Building Information Modelling - BIM). Esta mostra-se como uma das novidades mais promissoras dentro do mercado de Arquitetura, Engenharia e Construção (AEC). No Brasil, pode-se observar um cenário propício a investigação, pesquisa e discussões sobre o uso desta tecnologia na gestão de projetos e obras. Assim, o objetivo foi a realização de um diagnóstico identificando o atual cenário nacional de uso da tecnologia BIM e os ganhos reais do produto final. $\mathrm{O}$ trabalho caracteriza-se como um estudo indutivo, aplicado, descritivo e quantitativo que adotou como metodologia principal, além da revisão bibliográfica, a simulação de um levantamento tipo survey. A coleta de dados foi realizada por meio de um formulário construído e disponibilizado em endereço eletrônico preenchido pelos respondentes. Assim, a partir de indicadores e dados levantados do arcabouço legal e normativo no Brasil foi possível realizar pesquisa de campo sobre o uso do BIM em escritórios de projeto, construtoras e instituições de ensino. A realização do diagnóstico propiciou concluir que $67,21 \%$ dos entrevistados desconhecem ou apenas leram sobre o uso da tecnologia e que apenas $13,11 \%$ tem a mesma totalmente implantada. $\mathrm{O}$ reconhecimento de ganhos reais, não apenas do produto final, mas de todas as etapas do processo fazem com que $96 \%$ dos entrevistados que ainda não utilizam a tecnologia tenham perspectivas de sua utilização.

Palavras-chave: Modelo de Informação da Construção; Gestão de Projetos e Obras; Sistemas Colaborativos. 


\begin{abstract}
In the construction sector, every day new solutions in methods, tools, processes and concepts appear. Their use, as far as possible, allow companies to stay active and competitive. After presenting solutions of Enterprise Resources Planning (ERP), the area of Information Technology (IT) brings Building Information Modeling (BIM) to the construction industry. This is one of the most promising innovations in the Architecture, Engineering and Construction (AEC) market. In Brazil, one can observe a scenario conducive to research, research and discussions on the use of this technology in the management of projects and works. Thus, the objective was to carry out a diagnosis identifying the current national scenario of use of BIM technology and the real gains of the final product. The work is characterized as an inductive, applied, descriptive and

quantitative study that adopted as the main methodology, besides the bibliographic review, the simulation of a survey type survey. The data collection was done through a form built and made available in an electronic address filled out by the respondents. Thus, from the indicators and data gathered from the legal and normative framework in Brazil, it was possible to carry out field research on the use of BIM in project offices, construction companies and educational institutions. The diagnosis made it possible to conclude that $67.21 \%$ of the interviewees do not know or only read about the use of the technology and that only $13.11 \%$ have it fully implemented. The recognition of real gains, not only of the final product, but of all stages of the process, means that $96 \%$ of the interviewees who do not use the technology have prospects for their use.
\end{abstract}

Keywords: Construction Information Model; Management of Projects and Works; Collaborative Systems.

\footnotetext{
${ }^{1}$ Institutos Superiores de Ensino do CENSA - ISECENSA - Alunos Voluntários de Iniciação Científica PROVIC/ISECENSA - Rua Salvador Correa, 139, Centro, Campos dos Goytacazes, RJ, CEP: 28035-310, Brasil;

${ }^{2}$ Pesquisador do Laboratório de gestão de Projetos e Obras - LAGESPO/ISECENSA

(*) e-mail: mauriciodovale@gmail.com

Data de recebimento: 14/08/18. Aceito para publicação: 20/12/18 .
} 


\section{INTRODUÇÃO}

Nas últimas décadas, o avanço tecnológico dos computadores, software, smartphones e internet impactou tanto nas pessoas quanto nas organizações. Percebe-se a crescente quantidade e velocidade das informações geradas e disponibilizadas em ambientes reais e virtuais. Hoje, manter-se "atualizado" não é apenas ser detentor da mais avançada tecnologia e/ou de possuir mais informações, mas sim requer a garantia da qualidade e segurança destas, além de como aplicá-las. Nas organizações, em particular, requer o contínuo aprendizado e adaptação aos conhecimentos de processos gerenciais e em especial na formação das pessoas.

Para Nascimento, Laurindo e Santos (2003) depois de apresentar soluções de Enterprise Resources Planning (ERP), a Tecnologia da Informação (TI) trouxe avanços para diversos setores da economia. O setor da construção civil e a Modelagem da Informação da Construção (Building Information Modelling - BIM) surgiu como resultado de pesquisas científicas de países mais tecnologicamente desenvolvidos na década de 70. A necessidade era melhorar a tomada de decisão em vista a crescente quantidade de informações disponíveis e as novas exigências e especialidades esperada no mercado daqueles países, como por exemplo, as relacionadas à segurança, certificações ambientais, sustentabilidade, conforto, otimização de processos, gerenciamento de projetos, entre outros.

Desta forma, no campo da TI, a complexidade de investir nas equipes das organizações tem sido o maior desafio na atualidade, pois requer flexibilidade e mudanças na forma de pensar e agir dos profissionais atuantes no processo (idem). Assim uma vez superada essa barreira, novas oportunidades e novos avanços podem ser visualizados, pois via de regra, as tecnologias inovadoras trazem vantagens competitivas fáceis de serem identificadas.

No setor da construção civil, a cada dia surgem novas soluções em métodos, ferramentas, processos, conceitos, entre outros, e cabe aos envolvidos utilizá-las à medida do possível para conseguirem se manter competitivas (FORMOSO et al, 1999). Assim o BIM surge como uma importante ferramenta para analisar e facilitar o acesso a essas inovações. No entanto, é preciso que as empresas e profissionais estejam sempre avançando tecnologicamente, no ritmo certo, dentro das suas capacidades, mas sistematicamente evoluindo técnicas e hábitos dos seus colaboradores.

De fato, a indústria da construção está passando, ainda que de forma lenta e progressiva, por uma mudança de paradigma com a introdução dos conceitos e tecnologias do Modelo de Informação da Construção, ou simplesmente BIM. O conceito desta, mostra-se como uma das novidades mais promissoras dentro do mercado de Arquitetura, Engenharia e Construção (AEC). Este conceito envolve o gerenciamento de informações, através de softwares para desenvolvimento de projetos, desde a fase inicial do mesmo, criando um modelo digital que segue por todas as suas etapas abrangendo todo o ciclo de vida da edificação.

Recentemente, as construtoras de médio e pequeno porte passaram pela implantação dos softwares ERPs, que de maneira geral, exigiu bastante investimento de tempo, organização e padronização nas empresas. No entanto, percebeu-se que muitas foram as empresas nas quais a implantação deu-se apenas no âmbito do sistema de finanças (suprimentos, contabilidade e

Persp. Online: exatas \& eng., Campos dos Goytacazes, 23 (08) 48-62 - 2018

seer.perspectivasonline.com.br 
financeiro) dos empreendimentos, ficando em segundo plano o planejamento e a gestão da qualidade, conforme afirmam Nascimento, Laurindo e Santos (2003). Isso se dá pela dificuldade de geração e organização das informações em outras áreas.

Tais softwares, no mercado da AEC, surgem como fruto da evolução do Desenho Auxiliado pelo Computador (Computer Aided Design - CAD). A diferença básica, é que no BIM o mais importante não são desenhos como no CAD, mas sim, as informações que se apresentam de forma parametrizada. Esta parametrização aparece em softwares de bases de dados digitais (Autodesk Revit, ArchiCAD, MicroStation, VectorWorks e outros) que contemplam todos os aspectos a considerar numa edificação (SCHEER et al, 2007). Desta forma, o projeto é concebido e elaborado em um modelo virtual 3D ou maquete eletrônica, onde toda e qualquer alteração realizada neste resulte em alterações automáticas em todos os demais documentos que fazem parte do projeto, como cortes, elevações, vistas internas e de fachadas, quadro de áreas, tabelas de totalização de materiais e todo tipo de informação necessária a construção.

Portanto, pode-se afirmar conforme Eastman et al (2008) que são duas as principais "tecnologias" presentes no BIM que o diferenciam dos sistemas de CAD tradicionais, a saber: modelagem paramétrica e interoperabilidade.

A modelagem paramétrica permite representar os objetos por parâmetros e regras associados à sua geometria, assim como, incorporar propriedades não geométricas e características a esses objetos. Vale destacar que modelos de construção baseados em objetos paramétricos possibilitam a extração de relatórios, checagem de inconsistências de relações entre objetos e incorporação de conhecimentos de projeto, a partir dos modelos. Já a interoperabilidade é uma condição para o desenvolvimento de uma prática integrada. $\mathrm{O}$ uso desta com times de colaboração é possível com a integração da informação entre aplicativos computacionais, utilizados por diferentes profissionais de projeto.

Portanto, pode-se afirmar que entre as muitas vantagens que uma plataforma BIM oferece em comparação com o CAD tradicional, destacam-se: Rapidez na apresentação e entrega do projeto; Economia de tempo e custos na execução; Melhor coordenação do projeto e da execução da obra; Maior produtividade e redução de trabalho; Foco e ênfase no design; Rapidez nas revisões e alterações; Maior eficiência no controle de planilhas de materiais e cronogramas físico-financeiros.

Assim, os empreendimentos de construção civil normalmente são marcados pelo envolvimento de grande quantidade de profissionais e, em muitos casos, há diferentes especialistas envolvidos a cada projeto. Outra característica nestes empreendimentos é a variabilidade das informações a cada novo empreendimento, por serem executados em locais, condições, recursos e prazos diferentes.

Segundo Mendes Jr (1999), para se desenvolver construções mais ousadas, com metas mais rígidas de custo, por exemplo, as informações que fluem durante as fases de projeto e de obras precisam estar mais organizadas. Sendo esta a base para que as soluções entregues por cada um dos envolvidos sejam melhores, ou seja, mais eficientes, de menor risco, de melhor eficiência e eficácia, de melhor facilidade de aplicação na obra, entre outras. Além disso, muitas destas soluções acabam por afetar outras disciplinas do projeto, impactando nas

Persp. Online: exatas \& eng., Campos dos Goytacazes, 23 (08) 48-62 - 2018

seer.perspectivasonline.com.br 
soluções de outros profissionais da equipe, exigindo discussões e comprometimento de todos os envolvidos nos mais diversos níveis, ampliando ainda mais a a necessidade da colaboração.

Para Eastman et al (2008, p.13), o BIM é uma tecnologia de modelagem e um grupo associado de processos para produção, comunicação e análise do modelo de construção. Portanto, seu conceito, de acordo com esta definição, envolve tecnologias e processos que devem ser usados na produção, comunicação e análise dos modelos de construção.

Desta forma, a tecnologia BIM apresenta como meta, a busca por uma prática de projeto integrada, num sentido em que todos os participantes da AEC convirjam seus esforços para a construção de um "modelo único" de edifício. Assim, a utilização de uma prática baseada no BIM pode ter um papel decisivo na melhoria das fases do projeto, auxiliando na geração de propostas coerentes com as solicitações dos clientes, na integração dos projetos, entre si e com a construção, na redução do tempo e do custo da construção, assim como no processo de manutenção durante seu ciclo de vida.

Jacoski (2004) afirma que será o fim dos processos de projeto em que cada envolvido entrega sua parte (sua solução) baseada essencialmente em um plano de necessidades. Mas sim, o início dos processos de projeto colaborativo nas quais as soluções e necessidades são decididas de forma conjunta. Assim, colaborar é contribuir com as melhores soluções para um objetivo compartilhado, obtendo-se o melhor de cada um dos profissionais envolvidos neste. Por fim, todos contribuem com seus esforços, sendo a meta comum atingida pela soma dos objetivos de cada envolvido.

Portanto, é nesse contexto que surge o modelo computacional, cujo objetivo é conter todos os dados atualizados do projeto onde devem ser geradas (modeladas) as informações para serem usadas pela equipe colaborativa. Tal cenário, faz com que a inserção do BIM venha provocando uma profunda reorganização no setor da AEC em todo o mundo. Assim, segundo Ferreira (2007), o BIM pode ser definido como "representação virtual das características físicas e funcionais de uma edificação, por todo o seu ciclo de vida, servindo como um repositório compartilhado de informações para colaboração". Desta forma, trata-se de uma inovação tecnológica radical de processo, alterando funções, responsabilidades e conteúdo de produtos ao longo de todo o ciclo de vida das construções.

Destaca-se que o impacto do BIM não se limita às edificações, mas alcança desde a indústria de produtos e materiais, passando pelos projetos e obras de edifícios, estradas e outros tipos de infraestrutura e se prolonga pela manutenção, desmonte e/ou reuso destas obras. Entretanto, o setor da construção de edifícios pode ser visto como elemento central deste processo de difusão, pois cria demanda para os demais setores e assim orienta a difusão e uso do BIM.

Como dito, a implantação do BIM ocorre por meio de um conjunto de aplicativos que se articulam em plataformas digitais, compostas por tecnologias interoperáveis de um mesmo, ou de diferentes fornecedores. Tais plataformas definem uma nova tecnologia de processo, de projeto e de gestão da operação. Portanto, o foco na integridade e consistência da informação evidencia que o uso desta tecnologia traz novos processos de comunicação entre os diferentes agentes do projeto. 
Desta forma, para Formoso (2001), enquanto no processo de projeto clássico imagina-se em 3D e representa-se em 2D, no projeto de BIM imagina-se em 3D e a representação é através de uma construção virtual comumente chamada de modelo. A representação em 2D, ainda necessária, é quase completamente automatizada, sendo complementada e integrada a bases de dados externas, vinculada com especificações de requisitos de desempenho e outras informações que no processo de projeto anterior eram desconectadas entre si.

Segundo Witicowski e Scheer (2012), no cenário internacional muitas instituições e governos têm investido nos últimos anos em pesquisas no uso da tecnologia BIM. Entre as organizações internacionais e os mecanismos que garantem sua implementação, pode-se citar: International Alliance of Interoperability (IAI), National Institute of Building Sciences (NIBS), Associated General Contractors of America (AGC), Construction to Operations Building Information Exchange (COBIE), General Service Administration (GSA), International Council for Research and Innovation in Building and Construction (CIB), entre outras. Já com relação aos órgãos governamentais, de alguns países, pode-se notar o incentivo ao uso maciço do BIM, seja por meio de investimentos em agências de pesquisa (como é o caso do Geological Society of America - GSA, nos Estados Unidos, da Senate Properties na Finlândia e da INNOVA na Europa), seja por meio de regulamentações para a construção, ou ainda, por meio de fóruns de discussões sobre o uso desta tecnologia.

Na Europa, em especial por ser percursor na vanguarda desta tecnonologia, o mercado da AEC tem um importante papel na Economia Europeia, correspondendo aproximadamente a $10 \%$ do PIB e gerando 20 milhões de empregos, além de ser uma grande consumidora de produtos intermediários e de serviços relacionados. Com isso, as autoridades políticas da União Europeia (UE) reconhecem que o aperfeiçoamento da indústria da construção é importante não apenas para o crescimento e a geração de empregos, mas também para a sustentabilidade do próprio setor (European Commission, 2012).

No cenário nacional, a área acadêmica foi a primeira a demonstrar interesse sobre a tecnologia BIM. Em 1996, foram defendidas as primeiras dissertações no tema, na Universidade Federal Fluminense (UFF). No ano de 2000, teve início o projeto de Classificação e Terminologia para a Construção (CDCON), em resposta a um Edital da linha HABITARE, da FINEP, Financiadora de Estudos e Projetos, apoiado também pelo CNPq Conselho Nacional de Desenvolvimento Científico e Tecnológico - MCT - Ministério da Ciência e Tecnologia - e ANAMACO - Associação Nacional dos Comerciantes de Material de Construção (ANDRADE e RUSCHEL, 2009). De fato, o projeto do CDCON veio fortalecer a discussão das aplicações de Tecnologia de Informação na construção, tendo sido o embrião de outros grupos de pesquisa que se constituíram desde então.

Atualmente, boa parte da pesquisa sobre o BIM nas universidades se articula através de laboratórios da Rede BIM Brasil (www.redebimbrasil.org.br), constituído por um conjunto de grupos de pesquisa de universidades brasileiras, a saber: Universidade Federal do Paraná (UFPR ); Universidade de São Paulo (USP); Universidade Federal Fluminense (UFF); Universidade Federal da Bahia (UFBA); Universidade Estadual de Campinas (UNICAMP); Universidade Federal de Viçosa (UFV ); Universidade Federal do Rio Grande do Sul (UFRGS); Universidade Presbiteriana Mackenzie (UPM); Universidade Federal do Ceará 
(UFC).

Souza, Filho e Amorim (2009) afirmam que o setor produtivo nacional reconhece a importância do uso da tecnologia BIM, porém reclama da mão de obra capacitada para trabalhar com tal tecnologia. Mesmo assim, acreditam ter seus escritórios trabalhando na nova plataforma em um horizonte de cinco a dez anos.

De fato, no Brasil as pesquisas sobre BIM são muito recentes. Trabalhos que abordam objetos paramétricos, interoperabilidade e colaboração digital estão presentes em diversos congressos e eventos nacionais a mais de uma década. Porém, publicações com pesquisas que abordam a terminologia BIM são bem recentes. Para Ruschel e Andrade (2013), as propostas em geral destes trabalhos abordam: Conceitos básicos do BIM; Seu uso no processo de projeto; Sua relação com a colaboração e interoperabilidade; E, a sua customização.

Portanto, o que se pode observar é que o cenário nacional é propício a investigação, pesquisa e discussões sobre o uso da tecnologia BIM na gestão de projetos e obras nos setores de Arquitetura, Engenharia e Construção (AEC).

Desta forma, constitui-se como objetivo geral a realização do diagnóstico identificando o atual cenário do uso da tecnologia BIM por estudantes, profissionais liberais e empresas. Para o alcance deste foram delineados os seguintes objetivos específicos, a saber: a) Analisar o cenário do uso da tecnologia BIM no Brasil no mercado da AEC, profissional e acadêmico; b) Realizar pesquisa de campo sobre o uso da tecnologia BIM em escritórios de projetos, construtoras e instituições de ensino; c) Evidenciar as razões para o aprendizado e/ou utilização da mesma, assim como os ganhos reais do produto final na gestão de projetos e obras nos setores da AEC.

\section{METODOLOGIA}

O estudo realizado caracteriza-se como método indutivo, onde a partir de dados levantados procurou-se argumentos para levar a conclusões mais amplas do que as premissas nas quais se basearam a proposta. Assim, quanto a sua finalidade o mesmo apresenta-se como aplicada uma vez que busca gerar conhecimento para a aplicação prática e dirigida a solução dos problemas formulados quanto ao uso da tecnologia BIM no mercado da AEC.

No que se refere aos seus objetivos, o estudo é descritivo e tem como proposta o levantamento de dados a partir de questionários estruturados que visaram descobrir a existência de associações entre as variáveis analisadas na amostragem estabelecida. Para tanto, adotou-se uma abordagem quantitativa quanto ao problema de pesquisa. Esta abordagem permite compreender os fenômenos através da coleta de dados numéricos, que apontarão preferências, comportamentos e opiniões dos indivíduos que pertencem ao grupo amostral selecionado para a pesquisa em questão. 
Quanto a amostragem, a mesma apresenta-se como aleatória e não-probabilística. Foi selecionada uma amostra de 150 (cento e cinquenta) respondentes, a fim de generalizar os resultados para uma população atuante no mercado da AEC. Com isso, o delineamento da pesquisa foi bibliográfico buscando a fundamentação teórica para a realização da pesquisa de campo. Esta pesquisa foi realizada sobre o uso da tecnologia BIM em escritórios de projetos, construtoras e instituições de ensino. A coleta de dados foi realizada por meio de um formulário construído e disponibilizado em endereço eletrônico aplicado aos respondentes do grupo amostral.

A elaboração do questionário foi estruturada em 10 (dez) grupos de perguntas que foram direcionados a partir do Grupo 01 (um) que tratou da caracterização do respondente. A partir da caracterização deste as perguntas eram direcionadas automaticamente para os demais grupos em função do conhecimento ou não da tecnologia BIM, conforme pode ser observado na Figura 01.
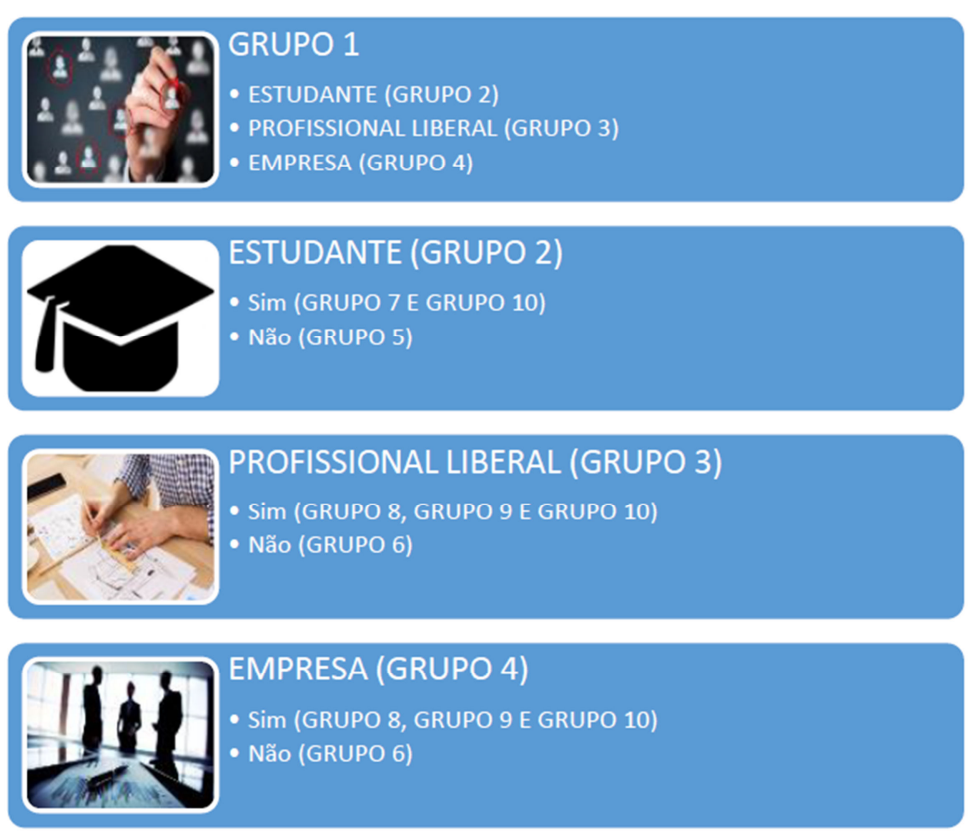

Figura 01: Estrutura do questionário em função da caracterização dos respondentes.

A partir deste primeiro momento as perguntas dos Grupos 02 (dois), 03 (três) e 04 (quatro) trataram respectivamente sobre as identificações do curso, do profissional e da empresa. Uma vez caracterizado estes três segmentos, os respondentes eram questionados sobre o uso da tecnologia BIM. As respostas tinham as opções de "sim" ou "não" que os levariam aos demais grupos de perguntas finalizando assim a pesquisa de campo através da aplicação do questionário.

A ferramenta utilizada para elaboração do questionário foi o Google Forms um instrumento de coleta e análise de dados que pertence ao pacote de serviços do Google e é disponibilizado gratuitamente. Esta ferramenta funciona de maneira on-line possibilitando o acesso a partir de computadores, tablets e smartphones. Tal ferramenta possibilita, ainda, a criação, formatação, divulgação e tabulação dos dados de uma pesquisa de maneira rápida, fácil, e segura para o pesquisador. 


\section{RESULTADOS}

Quanto a caracterização da população amostral dos 150 (cento e cinquenta) questionários aplicados aos respondentes foram descartados 28 (vinte e oito). Estes aconteceram em função de ajustes complementares e necessários na elaboração do formulário. Assim dos 122 (cento e vinte e dois) questionários válidos a população amostral, conforme pergunta do Grupo 01 (um), os respondentes foram caracterizados conforme a Tabela 01.

Tabela 01: Caracterização do respondente.

\begin{tabular}{|c|c|c|}
\hline GRUPOS & TIPO & QUANTIDADE \\
\hline Grupo 02 & Estudante & 41 \\
\hline Grupo 03 & Profissional Liberal & 45 \\
\hline Grupo 04 & Empresa & 36 \\
\hline \multicolumn{2}{|c|}{ TOTAL } & 122 \\
\hline
\end{tabular}

Quanto ao uso da tecnologia BIM, o Grupo 02 (dois) relacionado aos estudantes de arquitetura e urbanismo, engenharia civil e ambiental apresentaram os seguintes resultados (Figura 02) quando analisados os 41 (quarenta e um) formulários respondidos.

\section{ESTUDANTES}

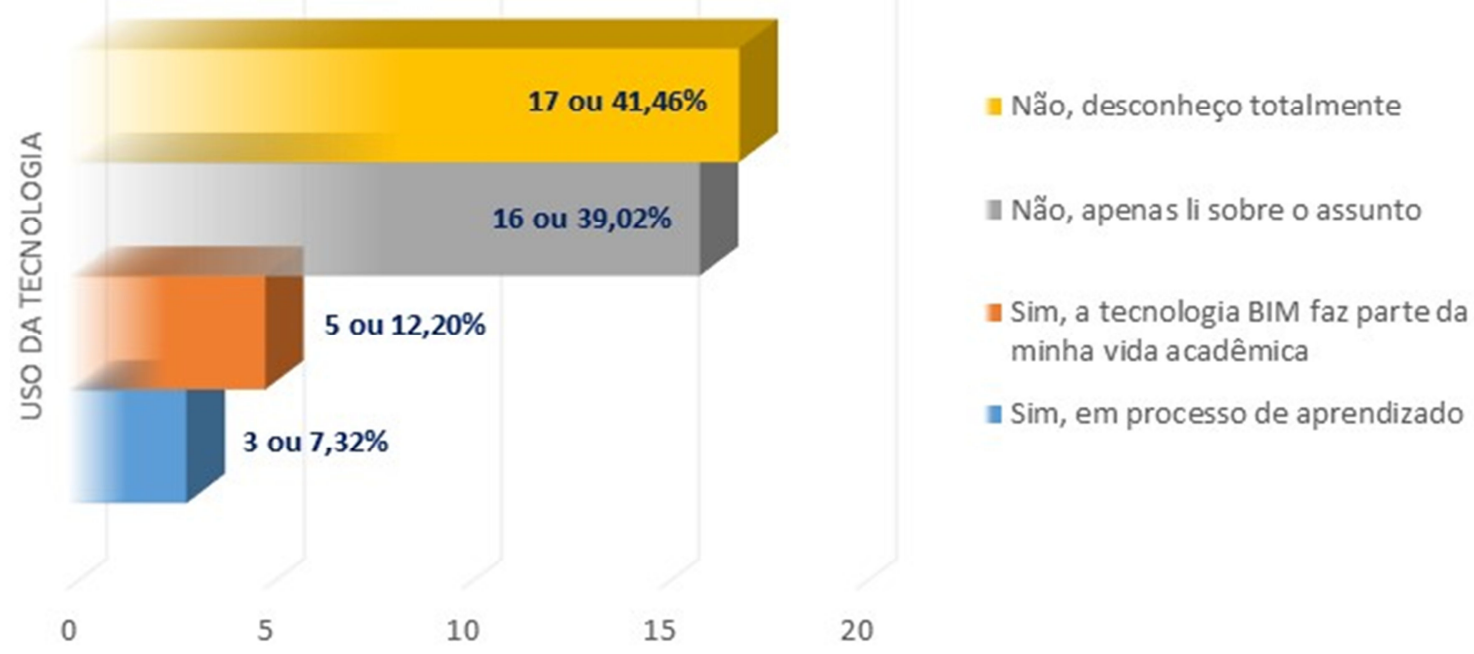

Figura 02: Avaliação dos estudantes quanto ao uso da tecnologia BIM.

Observa-se que a grande maioria $80,48 \%$ dos estudantes desconhecem ou apenas leram sobre a tecnologia BIM. Dos restantes, $12,20 \%$ tem o uso desta tecnologia na sua vida acadêmica e apenas $7,32 \%$ encontram-se em processo de aprendizado complementar a vida acadêmica. 
Já com relação ao Grupo 03 (três), onde foram avaliados os profissionais liberais quanto ao uso da tecnologia BIM, ficou evidenciada a necessidade de conhecimento de tal tecnologia para as atividades diárias da atuação profissional, conforme demonstrado na Figura 03.

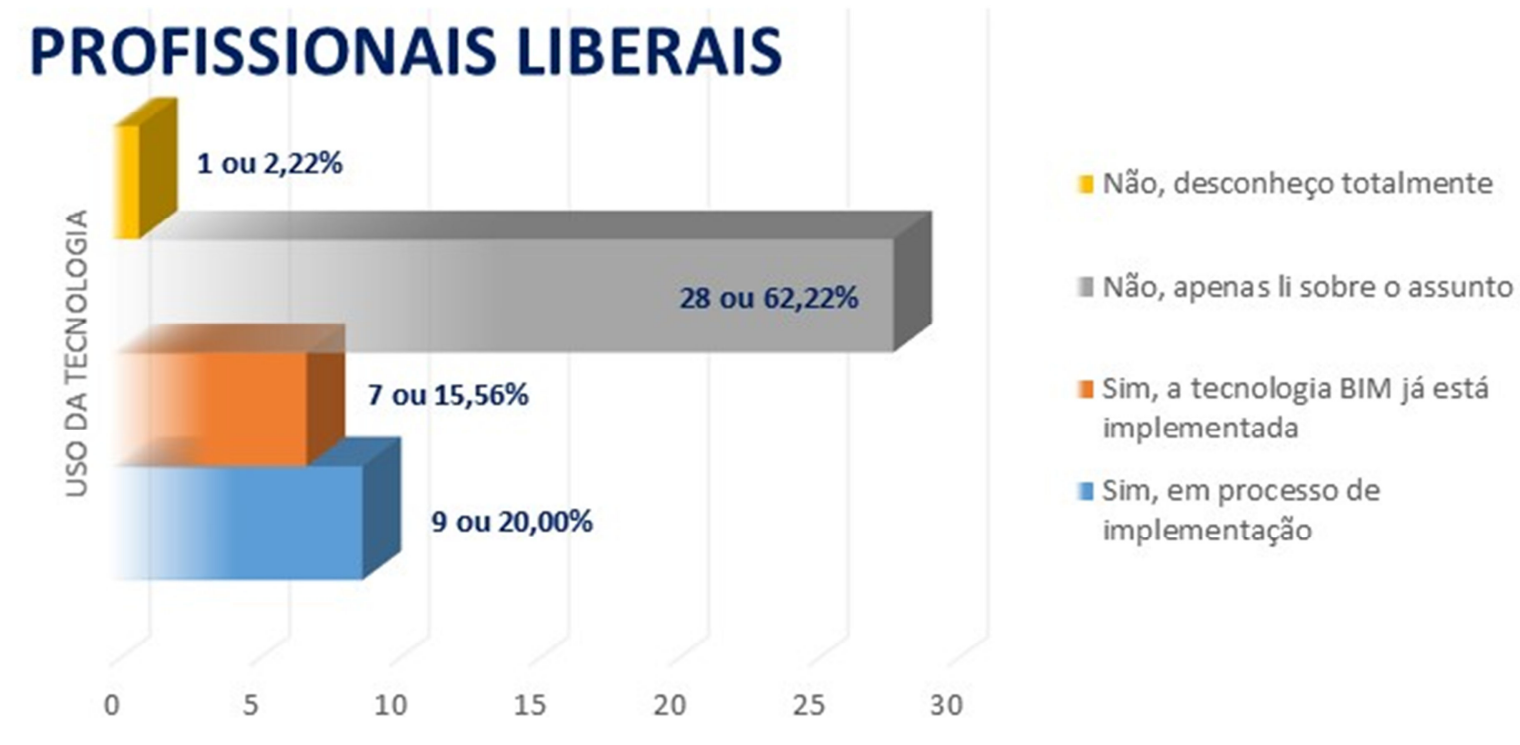

Figura 03: Avaliação dos profissionais liberais quanto ao uso da tecnologia BIM.

Assim, percebe-se que $35,56 \%$ destes profissionais que participaram da pesquisa interagem regularmente com tal tecnologia. Destes, observou-se que $15,56 \%$ já possuem a tecnologia BIM totalmente implementada e $20 \%$ estão em processo de implementação da tecnologia BIM em suas atividades profissionais. No entanto, a grande maioria $62,22 \%$ apenas leu sobre o assunto o que demonstra uma quantidade significativa de profissionais a absorverem o uso desta tecnologia em suas atividades profissionais diárias.

No cenário empresarial, que representa o Grupo 04 (quatro) desta pesquisa, percebe-se um aumento significativo no uso da tecnologia BIM. No entanto, os resultados demonstram, ainda, uma carência e necessidade de conhecimento por parte das empresas analisadas conforme pode ser observado na Figura 04. 


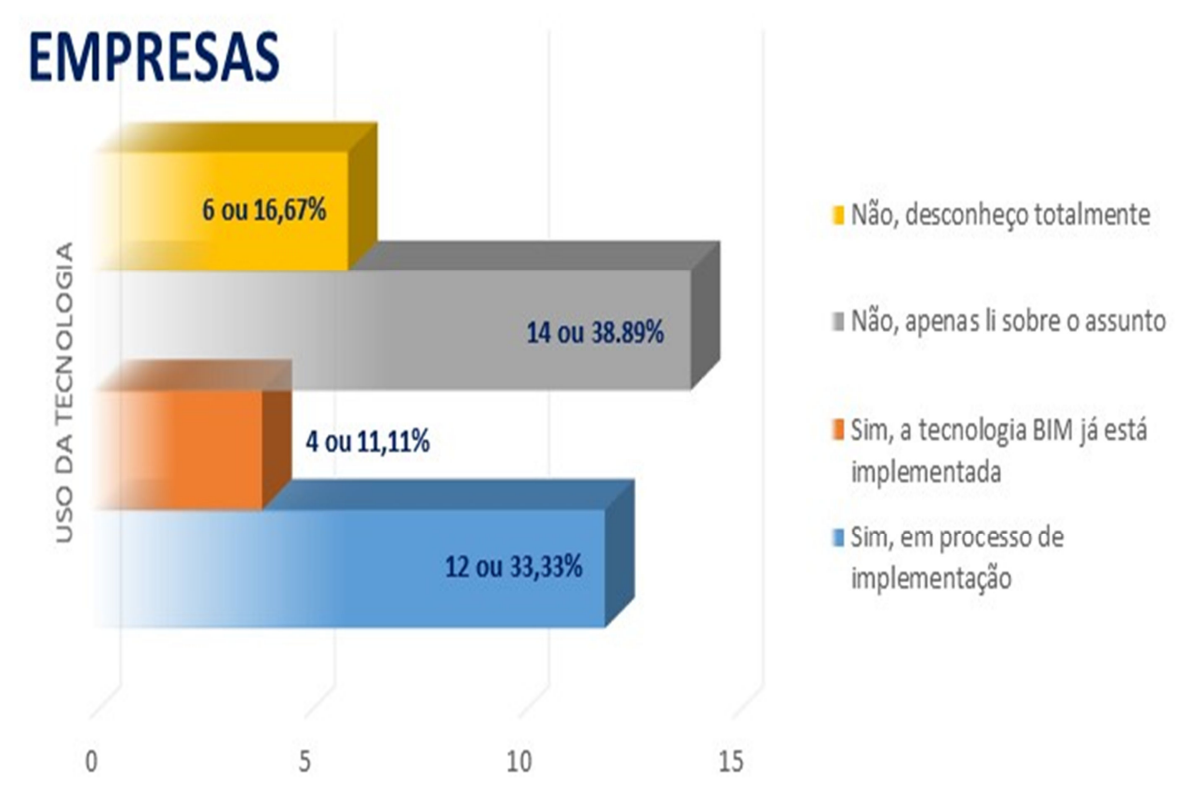

Figura 04: Avaliação das empresas quanto ao uso da tecnologia BIM.

Assim, observou-se que $44,44 \%$ das empresas que participaram da pesquisa interagem regularmente com tal tecnologia, o que representa um número superior aquele observado quanto aos profissionais liberais (35,56\%). No entanto, percebe-se que 55,56\% das empresas pesquisadas desconhecem ou apenas leram sobre o assunto. Resultado que evidencia um cenário propício a aquisição de conhecimento e aplicação do uso da tecnologia BIM nas atividades regulares destas empresas.

O resultado consolidado na análise destes Grupos (Grupos 02, 03 e 04) demonstram que $67,21 \%$ dos respondentes desconhecem ou apenas leram sobre o uso da tecnologia BIM, conforme observado na Figura 05. Nota-se que o Grupo 02 (dois) relacionado aos estudantes foi aquele que apresentou maior percentual de desconhecimento sobre o uso desta tecnologia, número que foi reduzido progressivamente quando analisados os profissionais liberais e empresas, Grupos 03 (três) e 04 (quatro) respectivamente. 


\section{CONSOLIDADO}

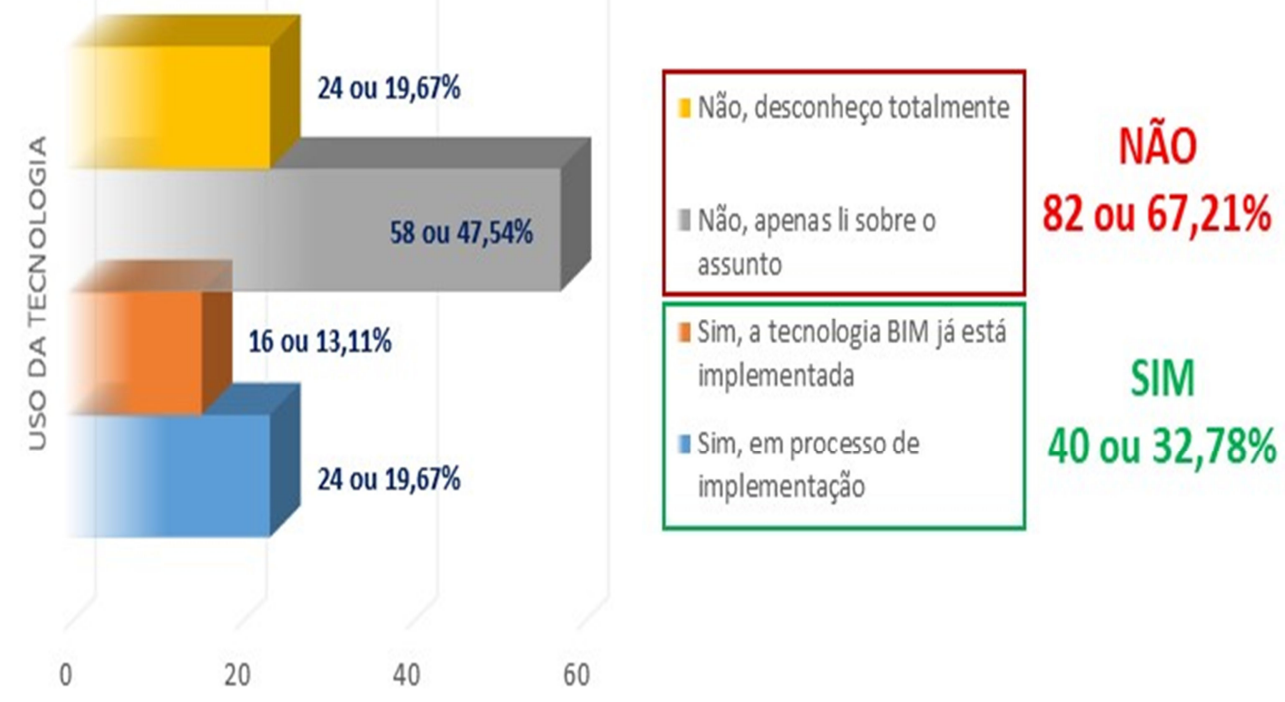

Figura 05: Resultado consolidado quanto ao uso da tecnologia BIM.

Portanto, os resultados consolidados na análise destes Grupos confirmam um cenário propício a investigação, pesquisa e aprendizado sobre o uso da tecnologia BIM na gestão de projetos e obras nos setores de Arquitetura, Engenharia e Construção (AEC).

Tais resultados se confirmam e tornam-se ainda mais relevantes quando analisados os resultados da pesquisa referente ao Grupo 10 (dez) que trata sobre as percepções e ganhos reais do produto final no uso da tecnologia BIM.

O Grupo 10 (dez), no formulário, corresponde as perguntas que visam analisar junto aos respondentes as questões relacionadas as percepções quanto ao uso da tecnologia BIM. Para tanto, foi utilizada a Escala Likert, onde os respondentes estabeleceram notas de acordo com o nível de importância atribuído aos parâmetros técnicos e as vantagens percebidas quanto ao uso da tecnologia. As notas atribuídas variavam em uma escala de 1 a 5, conforme o padrão Likert, onde: 1 corresponde a "nada importante"; 2 - "pouco importante"; 3 - "suficientemente importante"; 4 - "importante" e 5 - "muito importante".

Assim, sobre a análise dos parâmetros técnicos analisados no Grupo 10 (dez) a Figura 06 apresenta os resultados sobre a percepção dos respondentes quanto ao uso da tecnologia BIM. Para tanto, foram analisadas as seguintes variáveis, a saber: A) Produção e atualização de plantas, cortes, fachadas e detalhamentos de modo automático; B) Associação da volumetria, acabamentos e técnicas construtivas as planilhas de material, tempo de execução e custos; C) Comunicação e transferência de informações entre os participantes (equipe de projeto, fiscalização, empresas executantes e etc); D) Compatibilização e atualização dos mais variados projetos entre os participantes (projeto arquitetônico, estrutural, instalações e etc.). 


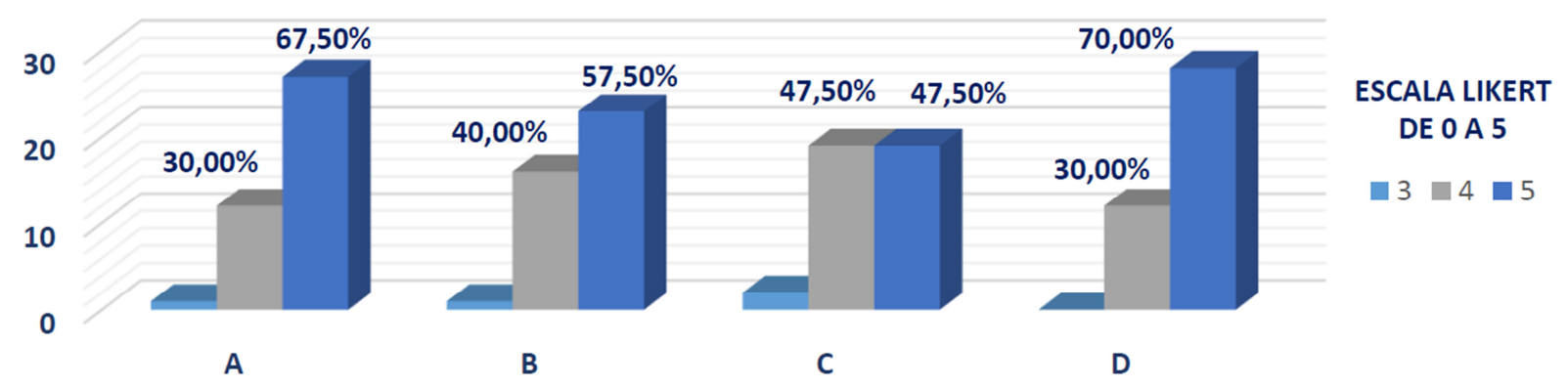

Figura 06: Importância atribuída ao uso da tecnologia BIM quanto aos parâmetros técnicos.

Destaca-se na análise destes parâmetros técnicos que mais de $90 \%$ das notas, dadas pelos respondentes, variaram entre 4 (quatro) e 5 (cinco) que para o padrão Likert compreende entre importante e muito importante. No entanto, vale ressaltar que nenhum dos parâmetros analisados recebeu notas abaixo de 3 (três) - suficientemente importante.

Ainda com relação a análise dos parâmetros técnicos avaliados neste Grupo 10 (dez), nota-se que três (A, B e D) dos quatros parâmetros obtiveram percentuais acima dos $50 \%$ para a Nota 5 - Muito Importante. O parâmetro "C", único que figurou abaixo dos 50\%, nesta nota, confirma a dificuldade da difusão desta tecnologia uma vez que trata dos aspectos de comunicação e transferência de informações entre os participantes (equipe de projeto, fiscalização, empresas executantes e etc).

No entanto, os parâmetros "A" e " $D$ " foram aqueles que apresentaram os maiores percentuais para a Nota 5 - Muito Importante. Tais parâmetros tratam respectivamente dos aspectos ligados a: Produção e atualização de plantas, cortes, fachadas e detalhamentos de modo automático; E, compatibilização e atualização dos mais variados projetos entre os participantes (projeto arquitetônico, estrutural, instalações e etc.)

Portanto, com relação a análise do nível de importância atribuído aos parâmetros técnicos, avaliados neste Grupo 10 (dez), fica evidente a tendência do uso da tecnologia BIM pelos estudantes, profissionais liberais e empresas. Confirmando-se os ganhos reais de produção, atualização e compatibilização técnica no desenvolvimento e gestão de projetos e obras no mercado da AEC.

Ainda no Grupo 10 (dez) e agora com relação a análise das vantagens percebidas quanto ao uso da tecnologia BIM os resultados são apresentados na Figura 07. Para tanto, foram analisadas as seguintes variáveis, a saber: A) Redução do tempo de projeto; B) Melhor representação e visualização do projeto, contribuindo para a redução de erros e omissões de projeto; C) Melhora na interoperacionalidade da relação entre os participantes e na identificação e/ou resolução entre os intervenientes da obra; D) Melhora na percepção e compreensão do projeto e desenvolvimento da obra com relação ao cliente final. 


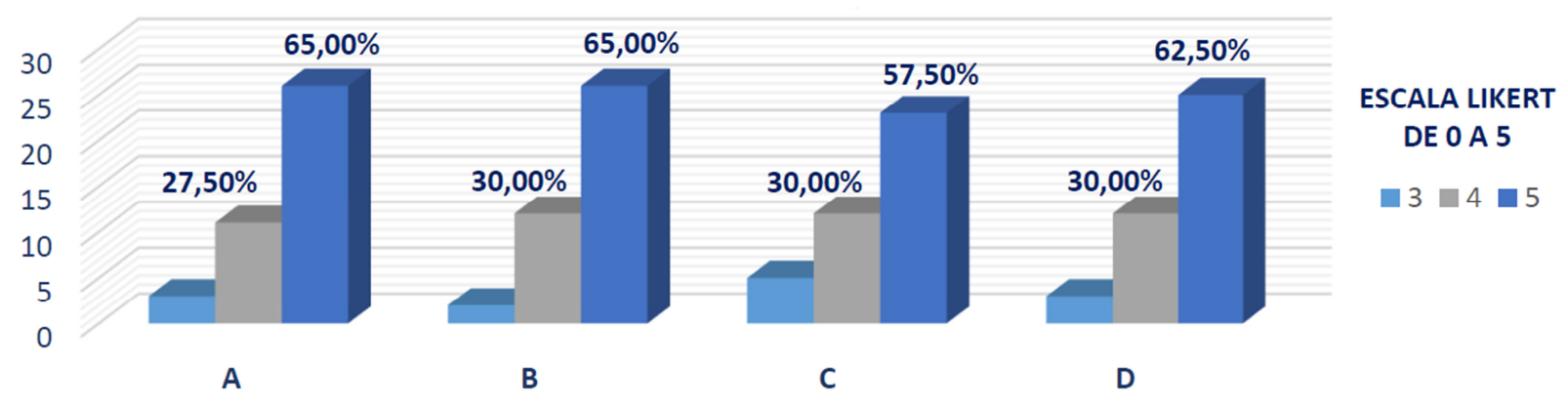

Figura 07: Importância atribuída as vantagens percebidas quanto ao uso da tecnologia BIM.

Destaca-se na análise da percepção destas variáveis que três (A, B e D) dos quatro parâmetros avaliados obtiveram mais de $90 \%$ das notas, dadas pelos respondentes, variando entre 4 (quatro) e 5 (cinco) que para o padrão Likert compreende entre importante e muito importante. O parâmetro " $C$ ", único desta análise, que não figurou nesta margem apresentou valor bem próximo de $87,50 \%$. No entanto, vale ressaltar que nenhuma das variáveis analisadas recebeu notas abaixo de 3 (três) - suficientemente importante.

Ainda com relação a análise das variáveis quanto as vantagens percebidas no uso da tecnologia BIM, realizada também neste Grupo 10 (dez), nota-se que os quatro parâmetros obtiveram percentuais acima dos $50 \%$ para a Nota 5 - Muito Importante. Portanto, ficou evidente os ganhos reais do produto final nesta análise por parte dos respondentes.

Desta forma, os resultados apresentados na análise do Grupo 10 (dez) quando relacionados aqueles demonstrados nos Grupos 2 (dois), 3 (três) e 4 (quatro) mostram um nicho de mercado propício a investigação, pesquisa e aprendizado sobre o uso da tecnologia BIM na gestão de projetos e obras nos setores de Arquitetura, Engenharia e Construção (AEC).

\section{CONSIDERAÇÕES FINAIS}

As fronteiras abertas pela Tecnologia da Informação (TI), em especial nas soluções de Enterprise Resources Planning (ERP), chegaram ao mercado de Arquitetura, Engenharia e Construção (AEC) com softwares que ampliaram os horizontes e estão promovendo uma mudança radical no setor. Tais softwares, no mercado da AEC, surgem como fruto da evolução do Desenho Auxiliado pelo Computador (Computer Aided Design - CAD). Assim, a introdução dos conceitos e tecnologias do Modelo de Informação da Construção, ou simplesmente BIM (Building Information Modelling) tem impulsionado e vem proporcionando uma mudança de paradigma neste segmento industrial.

Esta nova fase do setor da AEC marca o início dos processos de projeto colaborativo nas quais as soluções e necessidades são decididas de forma conjunta que é uma das premissas para o uso do BIM. Assim, esta nova fase vem levando gradativamente a redução dos processos de projeto em que cada envolvido entrega sua parte (sua solução) baseada essencialmente em um plano de necessidades. No entanto, no Brasil as pesquisas sobre BIM 
são muito recentes e demandam de avanços significativos.

Os resultados apontaram que $67,21 \%$ dos respondentes desconhecem ou apenas leram sobre o uso da tecnologia BIM. Outros $19,67 \%$ estão em processo de implantação e apenas $13,11 \%$ apresentam a tecnologia BIM totalmente implantada.

Já dos 32,78\%, respondentes, que sinalizaram conhecer a tecnologia BIM, sejam em fase de implantação $(19,67 \%)$ ou já com a tecnologia totalmente implantada $(13,11 \%)$ destacam pontos essenciais do uso desta tecnologia, a saber: a) Produção, compatibilização, atualização, comunicação e transferência de informações entre os participantes do projeto apresenta-se como um grande diferencial quando comparados ao sistema CAD tradicional; b) Ganho e vantagens no resultado do produto final quanto a redução no tempo de projeto, melhora na representação e visualização contribuindo para redução de erros e omissões de projeto, melhora na interoperacionalidade entre os participantes e percepção e compreensão do projeto e desenvolvimento da obra com relação ao cliente final.

Assim, a realização deste estudo veio confirmar o cenário propício a investigação, pesquisa, aprendizado e discussões sobre o uso da tecnologia BIM no Brasil, em especial, na gestão de projetos e obras nos setores de Arquitetura, Engenharia e Construção (AEC).

\section{REFERÊNCIAS}

ANDRADE, Max Lira Veras Xavier de; RUSCHEL, Regina Coeli. BIM: conceitos, cenário das pesquisas publicadas no brasil e tendências. SIMPÓSIO BRASILEIRO DE QUALIDADE DO PROJETO NO AMBIENTE CONSTRUÍDO, 2009, SÃO CARLOS, BRASIL, 2009. p. 602-613.

EASTMAN, C.; TEICHOLZ, P.; SACKS, R.; LISTON, K. BIM Handbook: a Guide to Building Information Modeling for Owners, Managers, Designers, Engineers, and Contractors. New Jersey:John Wiley \& Sons, 2008.

European Commission (2012). Communication from the Commission to the European Parliament and the Council: Strategy for the sustainable competitiveness of the construction sector and its enterprises. Brussels, 2012.

FERREIRA, Sérgio. Da engenharia simultânea ao modelo de informações de construção (BIM): contribuição das ferramentas ao processo de projeto e produção e vice-versa. PROCESSO DE PROJETO E PRODUÇÃO E VICE-VERSA. WORKSHOP BRASILEIRO DE GESTÃO DO PROCESSO DE PROJETOS NA CONTRUÇÃO DE EDIFÍCIOS, 7., 2007, CURITIBA, PR, 2007, Curitiba, PR, 2007. 6 p.

FORMOSO, C. T. (Org.). Planejamento e controle da produção em empresas de construção. Porto Alegre: Universidade Federal do Rio Grande do Sul - Núcleo Orientado para a Inovação da Edificação, 2001.

FORMOSO, C. T.; BERNARDES, M.; OLIVEIRA, L; OLIVEIRA, K. Termo de Referência para o Planejamento e Controle da Produção em Empresas Construtoras. Porto Alegre: 
Universidade Federal do Rio Grande do Sul, Programa de Pós-graduação em Engenharia Civil (PPGEC), 1999.

JACOSKI, C. A. A Integração da Comunicação em Projetos de Construção Utilizando Tecnologias da Informação. In: CONFERÊNCIA CONSTRUÇÃO, 2004, Portuga. FEUPPORTO/PORTUGAL, 2004.

MENDES JR, R. Programação da produção na construção de edifícios de múltiplos pavimentos. 196f. Tese (Doutorado em Engenharia da Produção). Departamento de Engenharia de Produção e Sistemas, Universidade Federal de Santa Catarina. Florianópolis, 1999.

NASCIMENTO, L. A., LAURINDO, F. J. B, SANTOS, E. T. A eficácia da TI na construção civil. São Carlos, 2003. III Sibragec 2003.

SCHEER, S.; ITO, A.; AYRES, C.; AZUMA, F.; BEBER, M. Impactos do Uso do Sistema CAD Geométrico e do Uso do Sistema CAD-BIM no Processo de Projeto em Escritórios de Arquitetura. In: VII Workshop Brasileiro de Gestão do Processo de Projeto na Construção de Edifícios, 2007, Anais... Curitiba - PR. VII Workshop Brasileiro de Gestão do Processo de Projeto na Construção de Edifícios, 2007.

SOUZA, Livia; FILHO, Arnaldo de Magalhães; AMORIM, Sérgio. Impactos do uso do BIM em escritórios de arquitetura: Oportunidades no mercado imobiliário. Tecnologia de Informação e Comunicação na Construção Civil. Rio de Janeiro, Brasil, 2009.

WITICOWSKI, Lilian C.; SCHEER, Sérgio. Some improvements for BIM based cost estimation. IN: 7TH INTERNATIONAL CONFERENCE ON INNOVATION IN ARCHITECTURE, ENGINEERING \& CONSTRUCTION, 2012, SÃO PAULO. Innovations in AEC 2012 Proceedings. São Paulo: Escola Politécnica/USP e CICE/Loughborough University, 2012. v. 1. p. 1-10. 